\title{
Hybrid Natural Orifice Transluminal Endoscopic Surgery with Sentinel Lymph Node Navigation for Deep Early Gastric Cancer in the Fundic Region
}

\author{
Yoon Suk Park ${ }^{1}$, Seong Hwan Kim ${ }^{1}$, Hee Yun Ryu', Young Kwan Cho ${ }^{1}$,Yun Ju Jo ${ }^{1}$, Tae il Son ${ }^{2}$ and Young Ok Hong ${ }^{3}$ \\ Departments of ${ }^{1}$ Internal Medicine, ${ }^{2}$ Surgery, and ${ }^{3}$ Pathology, Eulji General Hospital, Eulji University School of Medicine, Seoul, Korea
}

For patients refusing surgical treatment for deep early gastric cancer, hybrid natural orifice transluminal endoscopic surgery with sentinel lymph node navigation is a potential treatment option, particularly when the anatomic location of the cancer has low probability of lymph node metastasis. We report a case of deep early gastric cancer of the fundus beyond the endoscopic submucosal dissection indication that was treated by hybrid natural orifice transluminal endoscopic surgery with sentinel lymph node navigation. In a conventional approach, a total gastrectomy would have been needed; however, the patient refused surgical intervention. In this case, since the patient showed no positivity of the sentinel lymph node on intraoperative navigation, laparoscopic basin lymph node dissection was not performed. Hybrid natural orifice transluminal endoscopic surgery might be considered for specific regions such as the safety zone where lymph node metastases are less likely to occur. Clin Endosc 2016;49:298-302

Key Words: Natural orifice endoscopic surgery; Stomach neoplasms; Lymphatic metastasis

\section{INTRODUCTION}

The diagnostic rate of early gastric cancer (EGC) has remarkably increased because of developments of technology and cancer mass screening systems. Accurate diagnosis of EGC is crucial in improving the survival rate of gastric cancer. ${ }^{1}$ In recent years, the debate on expanding indications for endoscopic submucosal dissection (ESD) has been a subject of debate among clinicians, specifically regarding the treatment of cancers with lymph node metastasis (LNM). ${ }^{2}$ Yet few, if any, studies focus on the hybrid natural orifice transluminal endoscopic surgery (NOTES) technique which we believe to be the new alternative in EGC treatment. Gastrectomy and

Received: September 2, 2015 Revised: October 14, 2015

Accepted: October 14, 2015

Correspondence: Seong Hwan Kim

Department of Internal Medicine, Eulji General Hospital, Eulji University School of Medicine, 68 Hangeulbiseong-ro, Nowon-gu, Seoul 01830, Korea

Tel: +82-2-970-8297, Fax: +82-2-972-8621, E-mail: shkim@eulji.ac.kr

(cc) This is an Open Access article distributed under the terms of the Creative Commons Attribution Non-Commercial License (http://creativecommons.org/ licenses/by-nc/3.0) which permits unrestricted non-commercial use, distribution, and reproduction in any medium, provided the original work is properly cited. lymphadenectomy has been performed as the standard treatment of EGC beyond the scope of endoscopic resection. EGC beyond the classic ESD indications has the relatively high risk of LNM, which varies from $5.7 \%$ to $20 \%{ }^{3.5}$ Conversely, it suggests that in many cases surgeons may avoid gastrectomy with lymphadenectomy. Hybrid NOTES enables minimal surgical resection using the ESD technique, and a laparoscopic lymphadenectomy can also be performed simultaneously in cases of EGC with a risk of LNM. Thus, NOTES might be advised as a modified treatment for EGC. Like our case, EGC in the fundic area has a low probability of LNM. Moreover, the patient refused any surgical treatment. In this situation, hybrid NOTES might be considered as an optional treatment.

\section{CASE REPORT}

A 61-year-old man who underwent health examinations at an outside hospital was diagnosed with submucosal invasion of EGC and had been strongly advised to undergo total gastrectomy and lymphadenectomy. However, the patient visited our hospital for less invasive treatment such as an ESD. 

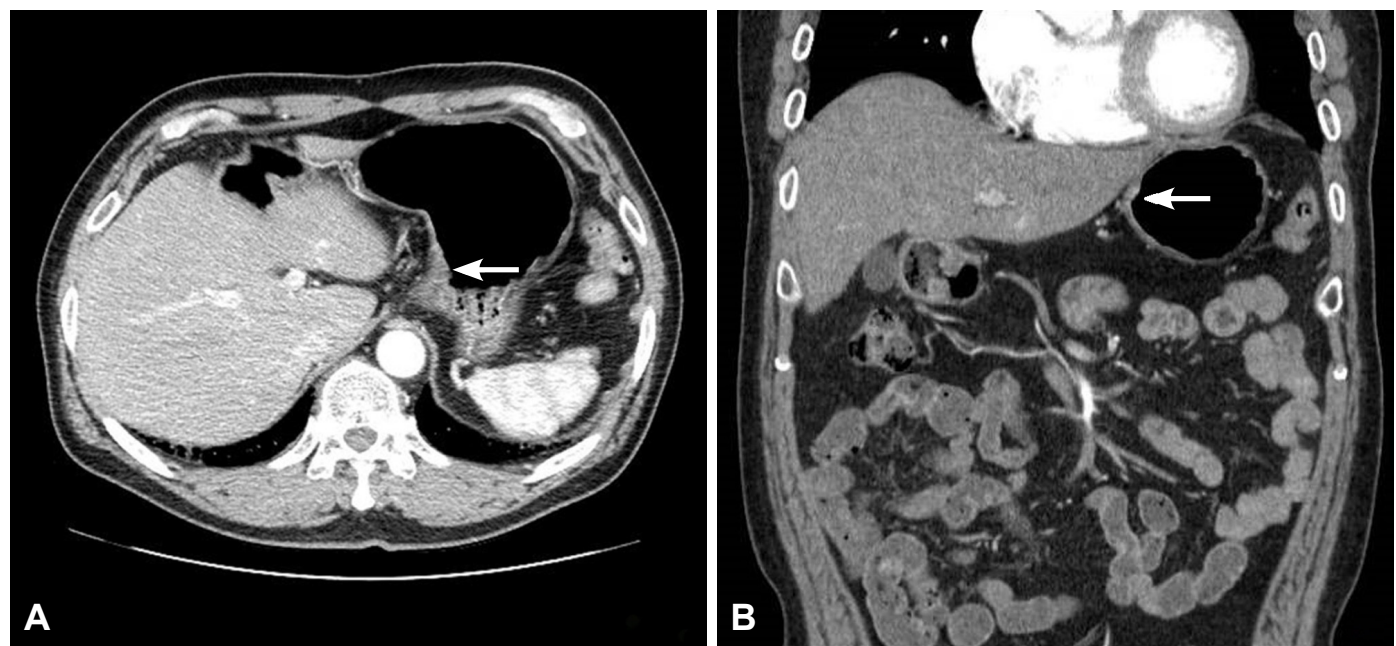

Fig. 1. (A, B) Transverse and coronal views of abdominal computed tomography scan showing mildly enhanced wall thickening (arrows) in the fundic area without perigastric lymphadenopathy.
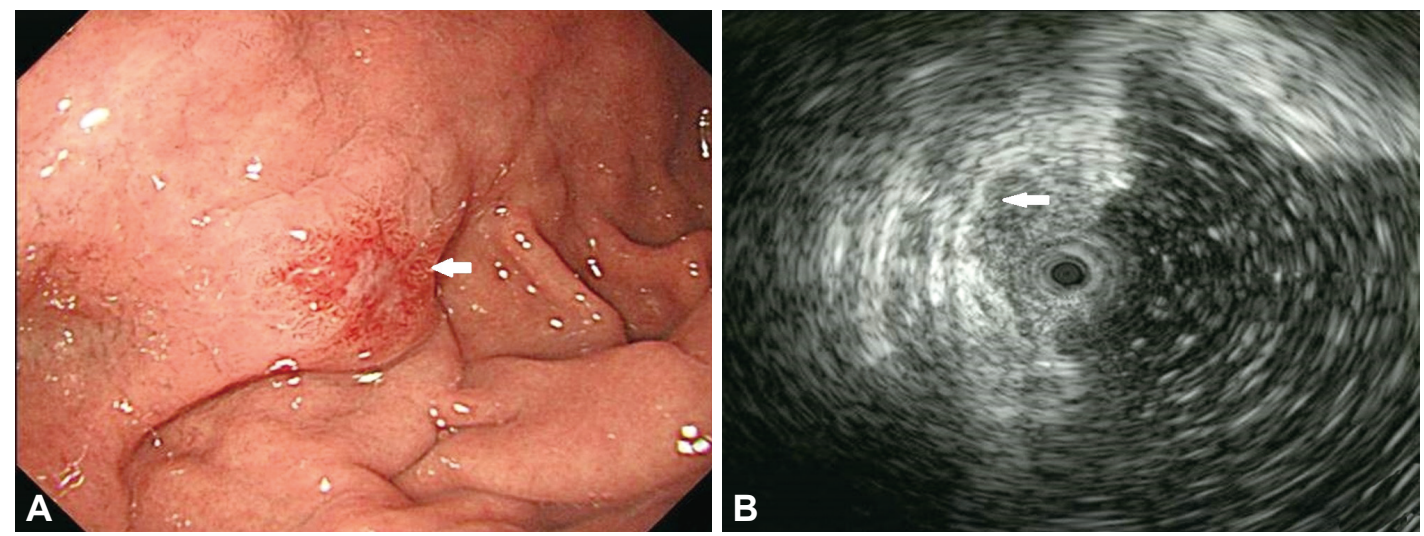

Fig. 2. (A) White light endoscopy showing an early gastric cancer lla lesion (arrow) with central ulceration at the fundic area. (B) Endoscopic ultrasonography revealed invasion (arrow) to the deeper submucosal layer.

Computered tomography (CT) demonstrated mild enhancing wall thickening (arrow) in the fundic area without perigastric fatty infiltration and lymphadenopathy (Fig. 1). White light endoscopy showed an approximately $1.5 \mathrm{~cm}$ EGC type IIa lesion (arrow) at fundus (Fig. 2A). Endoscopic ultrasonography showed findings suspicious for deep submucosal invasion (arrow) of the EGC (Fig. 2B). Total gastrectomy is typically required when deep invasion of the submucosal layer is suspected. However, the patient continued to refuse total gastrectomy. Ultimately, the decision was made to perform a hybrid NOTES procedure with sentinel lymph node (LN) navigation, which enabled us to perform an endoscopic full-thickness gastric resection and laparoscopic basin lymphadenectomy. Initially, we performed the chromoendoscopic observation of the lesion with indigo carmine spraying; we then marked a safety margin around the lesion and injected $1 \mathrm{cc}$ of indocyanine green at three different sites around the lesion (Fig. $3 \mathrm{~A})$. One camera port was placed at the umbilicus and four trocars were inserted in the upper left, upper right, lower left, and lower right abdominal quadrants. For 15 minutes after injection, we looked for the presence of a sentinel LN; however, the LN was not identified on laparoscopic view. Thus, we did not perform sampling of the sentinel LN. We then applied the ESD technique by making a circumferential incision as deep as the submucosal layer around the lesion (Fig. 3B-E). After completing the incision, we made a circumferential, endoscopic full-thickness resection around the lesion through the submucosal incision line. Subsequently, laparoscopic stapling was performed around two-thirds of the lesion through the circumferential, endoscopic full-thickness gastric resection incision line. ${ }^{6}$ The resected specimen was then retrieved. The procedure was completed by confirming the suture site and examining for any signs of bleeding (Fig. 3). The estimated total blood loss was less than $100 \mathrm{cc}$ during the surgery. Laparoscopic LN dissection was considered but was not performed (Fig. 4A), because no positive sentinel LN was confirmed (Fig. 4B). Pathologic examination of the resected full-thickness specimen demonstrated EGC type IIa, a moderately differen- 

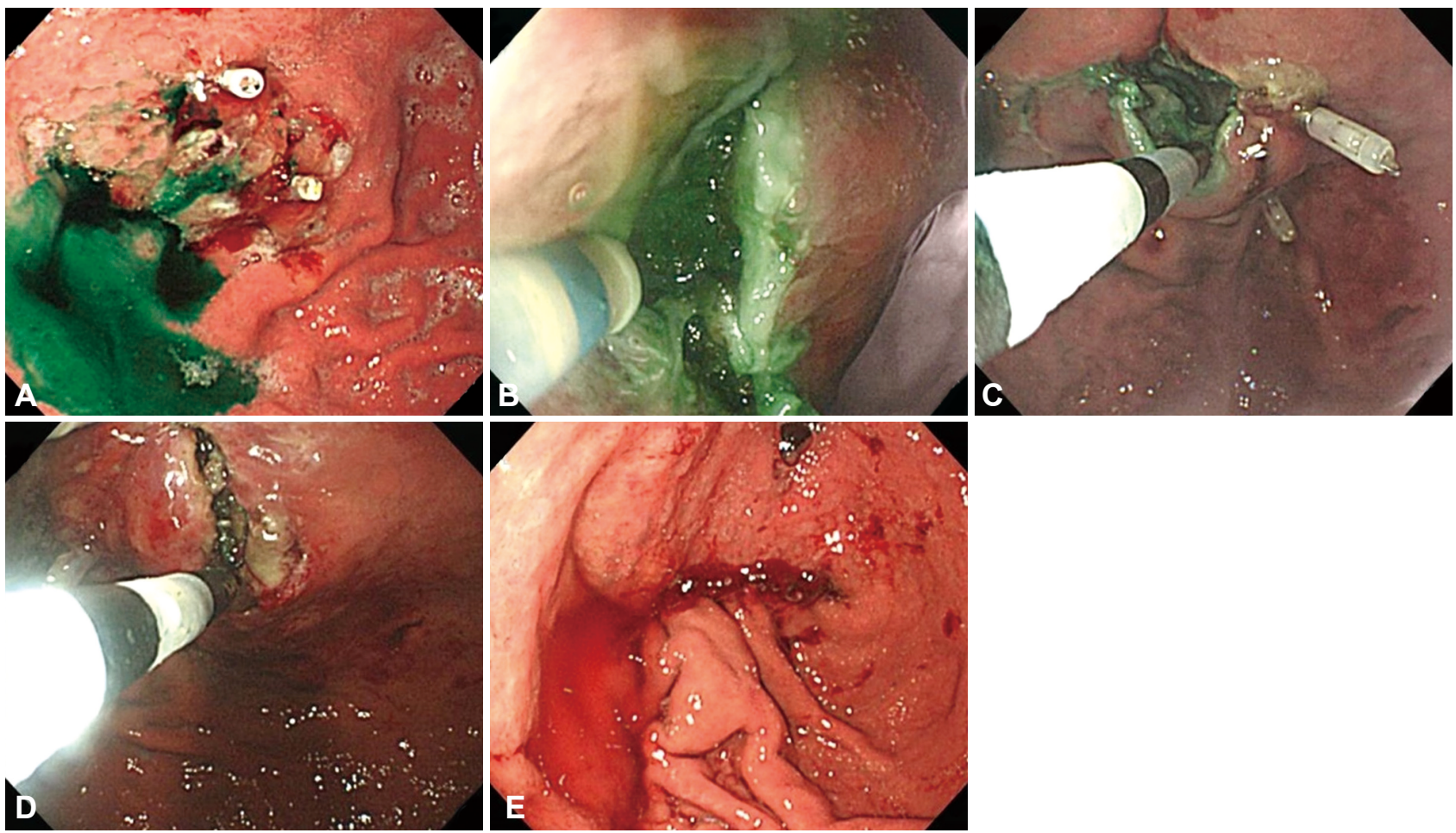

Fig. 3. (A) Perilesional indocyanogreen injections were performed intraoperatively. (B-D) Endoscopic full-thickness resection was performed by insulated knife. (E) Laparoscopic closure was performed by stapler.
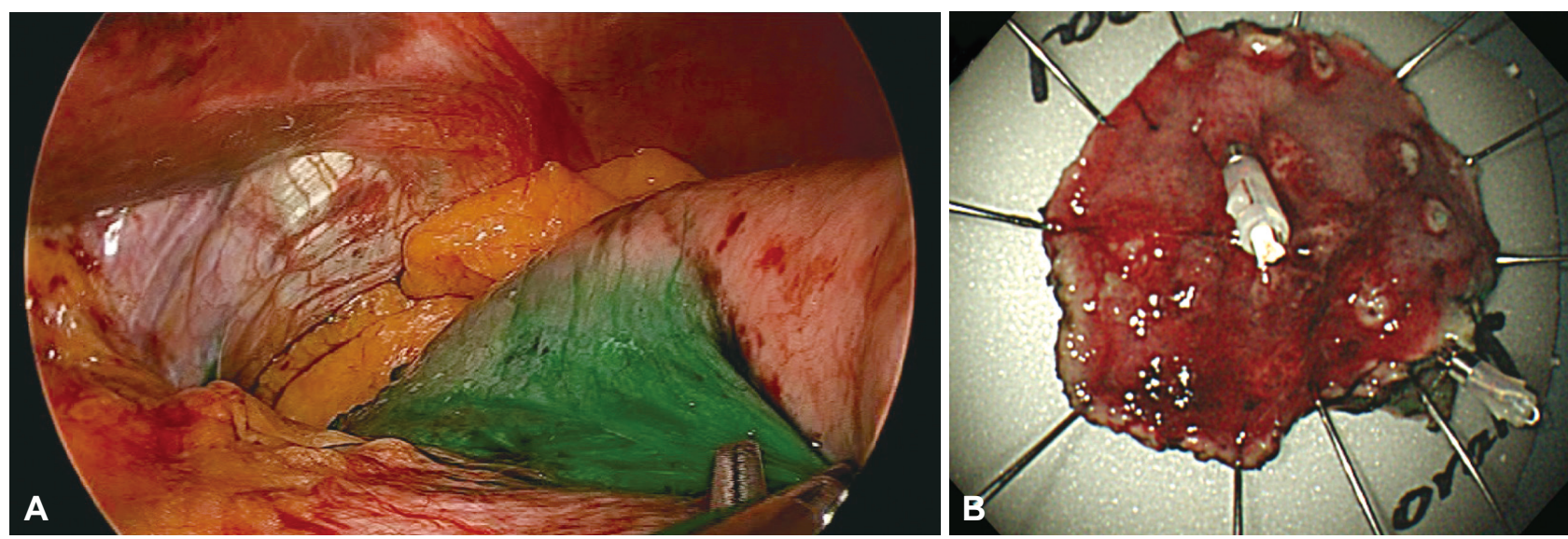

Fig. 4. (A) Laparoscopic view showing negativity of the sentinel lymph node after indocyanine green injection. (B) Resected full-thickness specimen revealed an adequate safety margin with all perilesional marking dots.

tiated adenocarcinoma with a mucinous component invading the 3rd submucosal layer, with a clear resection margin. No lymphovascular or perineural invasion was found (Fig. 5). No evidence of recurrence or complications has been noted; thus, far during 3 years of regular CT and endoscopic follow-up.

\section{DISCUSSION}

Minimally invasive surgery has been more and more indispensable with the increasing frequency of EGC. As the surviv- al rate of patients with EGC improves, the quality of life after surgery should also be evaluated with great care. Comparing the past surgical outcomes, the complication rate of gastrectomy has been significantly improved. However, a certain level of surgical complications is inevitable. ${ }^{7}$ For example, we cannot avoid post-gastrectomy syndromes such as dumping syndrome, oral intake restriction, and anemia following surgery. Minimally invasive modalities such as sentinel LN navigation, ESD, and NOTES are now in the limelight as a way to solve such problems. However, there are various ongoing debates over expanding the indications for minimally invasive treat- 

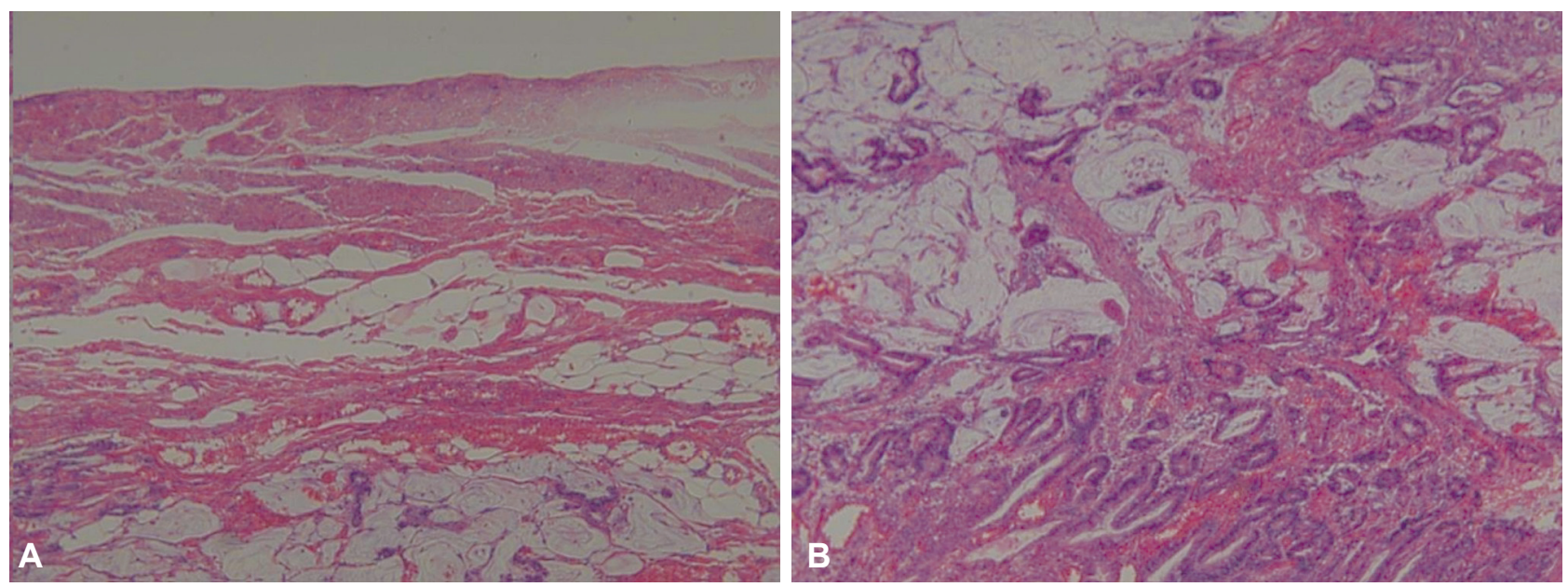

Fig. 5. Multiple well-formed tumor glands are noted in the mucosa and submucosa with abundant mucin pools (A, H\&E stain, $\times 100 ; B, H \& E$ stain, $\times 200)$.

ments such as ESD, because it is difficult to predict whether LNM exists. If deep submucosal invasion of EGC is suspected as in our case, we cannot be sure that there is no LNM, which is the single most important factor in prognosis of gastric cancer. Therefore, traditional minimally invasive modalities guarantee a cure; thus, the role of minimally invasive treatments such as ESD has been marginalized. We instead chose the hybrid NOTES technique with sentinel LN navigation, which offers the ability to make a full-thickness resection and selective metastatic LN dissection. The list of factors that are directly associated with LNM includes the depth and size of tumor and the presence of an ulcer. LNM occur less often in the upper-third portion of the stomach with a depth extending to the submucosal layer when compared to other areas. ${ }^{8-10}$ The presence of a safety zone with less lymphatic drainage in the fundus, which is a part of the upper-third portion of the stomach, makes EGC less likely to metastasize to nearby LN, as demonstrated in this case. ${ }^{9,10}$ Additionally, when compared to other areas of the stomach, the LN dissection range is smaller in the upper-third portion, making hybrid NOTES even more viable. ${ }^{10}$ In recent years, numerous studies have been conducted on sentinel node (SN) mapping. Until now, dye-based and radioisotope-based techniques have been the mainstay for $\mathrm{LN}$ detection. Using infrared ray beam or fluorescence imaging enabled physicians to increase the detection rates of traditional dye agents such as indocyanine green. ${ }^{11}$ In this case, $\mathrm{SN}$ mapping guidance by simple indocyanine green injection was used due to a lack of fluorescence imaging equipment in our facility. The indocyanine green fluorescence imaging technique allows the practitioner to visually determine LNM. A SN detection rate of up to $94.7 \%$ was confirmed in $\mathrm{T} 1$ and $\mathrm{T} 2$ gastric cancers through the $\mathrm{SN}$ mapping technique. ${ }^{12}$ However, this technique is currently still in the third phase of clinical trials and; thus, couldn't be a standard treatment. Despite the fact that long-term data needs to be accumulated before expanding indications for ESD and hybrid NOTES due to the possibility of recurrence through LNM, hybrid NOTES with sentinel LN evaluation could be carefully considered as a treatment option for EGC's beyond the expanded ESD indications that occur in the upper-third portion of the stomach, particularly the fundic area.

\section{Conflicts of Interest}

The authors have no financial conflicts of interest.

\section{REFERENCES}

1. Isozaki H, Okajima K, Yamada S, et al. Proximal subtotal gastrectomy for the treatment of carcinoma of the upper third of the stomach: its indications based on lymph node metastasis and perigastric lymphatic flow. Surg Today 1995;25:21-26.

2. Li H, Lu P, Lu Y, et al. Predictive factors for lymph node metastasis in poorly differentiated early gastric cancer and their impact on the surgical strategy. World J Gastroenterol 2008;14:4222-4226.

3. Gotoda T, Yanagisawa A, Sasako M, et al. Incidence of lymph node metastasis from early gastric cancer: estimation with a large number of cases at two large centers. Gastric Cancer 2000;3:219-225.

4. Hirasawa T, Gotoda T, Miyata S, et al. Incidence of lymph node metastasis and the feasibility of endoscopic resection for undifferentiated-type early gastric cancer. Gastric Cancer 2009;12:148-152.

5. Kwee RM, Kwee TC. Predicting lymph node status in early gastric cancer. Gastric Cancer 2008;11:134-148.

6. Cho WY, Kim YJ, Cho JY, et al. Hybrid natural orifice transluminal endoscopic surgery: endoscopic full-thickness resection of early gastric cancer and laparoscopic regional lymph node dissection: 14 human cases. Endoscopy 2011;43:134-139.

7. Draşovean R. Post-gastrectomy syndromes. Acta Medica Transilv 2012;2:201-203.

8. Ren G, Cai R, Zhang WJ, Ou JM, Jin YN, Li WH. Prediction of risk factors for lymph node metastasis in early gastric cancer. World J Gastroenterol 2013;19:3096-3107.

9. Boku T, Nakane Y, Okusa T, et al. Strategy for lymphadenectomy of gastric cancer. Surgery 1989;105:585-592.

10. Kitamura M, Arai K, Miyashita K. Indication and clinical results of 


\section{$C_{\text {cunncalenoscopr }}$}

proximal gastrectomy with special reference to lymph node metastasis in upper gastric cancers. J Jpn Pract Surg Soc 1991;52:1454-1460.

11. Symeonidis D, Koukoulis G, Tepetes K. Sentinel node navigation surgery in gastric cancer: current status. World J Gastrointest Surg
2014;6:88-93.

12. Tajima Y, Murakami M, Yamazaki K, et al. Sentinel node mapping guided by indocyanine green fluorescence imaging during laparoscopic surgery in gastric cancer. Ann Surg Oncol 2010;17:1787-1793. 